\title{
Multiple Access in Ultra- Wideband Communications Using Multiple Pulses and the Use of Least Squares Filters
}

\author{
F. U. Dowla, F. Nekoogar
}

This article was submitted to the "2003 IEEE Radio and Wireless Conference (RAWCON)." Boston, Massachusetts, August 10-13, 2003

Low

June 19, 2003

Livermore

National

Laboratory

This is a preprint of a paper intended for publication in a journal or proceedings. Since changes may be made before publication, this preprint is made available with the understanding that it will not be cited or reproduced without the permission of the author. 
This document was prepared as an account of work sponsored by an agency of the United States Government. Neither the United States Government nor the University of California nor any of their employees, makes any warranty, express or implied, or assumes any legal liability or responsibility for the accuracy, completeness, or usefulness of any information, apparatus, product, or process disclosed, or represents that its use would not infringe privately owned rights. Reference herein to any specific commercial product, process, or service by trade name, trademark, manufacturer, or otherwise, does not necessarily constitute or imply its endorsement, recommendation, or favoring by the United States Government or the University of California. The views and opinions of authors expressed herein do not necessarily state or reflect those of the United States Government or the University of California, and shall not be used for advertising or product endorsement purposes. 


\title{
Multiple Access in Ultra-Wideband Communications Using Multiple Pulses and the Use of Least Squares Filters
}

\author{
Farid Dowla and Faranak Nekoogar \\ Department of Applied Science, University of California Davis \\ Lawrence Livermore National Laboratory
}

94550 USA

Livermore, CA

\{dowla1, nekoogar1@1lnl.gov\}

\begin{abstract}
Multiple access (MA) in UWB communication has recently been studied in the context of multiple transmitted-reference short duration chirp pulses in the presence of additive white Gaussian noise (AWGN). The transmitted-reference (TR) receiver was extended in previous studies using multiple orthogonal pulses. Improved performance was observed in simulations by sampling the receiver autocorrelation function $(\mathrm{ACF})$ at both zero- and nonzero lags. Sampling of non-zero ACF lags of orthogonal pulses is a novel approach. Method of using least squares filtering techniques for further tolerance against noise and interference for the TR method is proposed.
\end{abstract}

\section{INTRODUCTION}

Since ultra-wideband (UWB) systems use short duration (sub-nano sec) pulses that spread their energy across a wide range of frequencies [1], pulse shaping and filtering is a rich area of study in this problem. This is particularly true for the TR modulation, a technique recently discussed by the by several researchers.

Recently, we have studied the multiple access techniques for the TR method for channelization of multiple users. In a multiple access UWB communications system, users transmit information independently and concurrently over a shared channel. The received signal is therefore a superposition of all user signals with added channel noise. There has been extensive research in separating multiple

This work was performed under the auspices of the U.S. Department of Energy by University of California Lawrence Livermore National Laboratory under contract No. W-7405-Eng-48. users in a multiple access UWB system using TDMA or CDMA Pulse Amplitude Modulation (PAM) and Pulse Position Modulation (PPM) techniques [4, 5, 6 and 7]. PAM modulation encodes the data bits based on different levels of power (amplitude) in short duration pulses. In PPM modulation, signals are pseudo randomly encoded based on the position of transmitted pulse trains by shifting the pulses in a predefined window in time. PPM transmitted signals are usually demodulated and recovered with template matching at the receiver. Conventionally, both of these modulation techniques use a single pulse shape to transmit and receive data for all users. One concern with using the same pulse shape for all channels is that multiple access interference (MAI) increases with the number of users. This is due to increased cross-correlation between similar pulses from various channels, raising thus the noise floor in such systems. To overcome high cross-correlation between similar pulses, Ghavami and Kohono in [10] have suggested the pulse shaping modulation using Hermite-based orthogonal pulses. Using multiple pulses with low crosscorrelation in multiple access systems reduces the MAI effect.

Others [8,9] have proposed the use of a symbol that consists of a pair of pulses (doublets) separated by a unique delay to represent data bits. The first pulse is fixed and the second pulse is modulated by data using opposite polarities to represent one or zero. This method has the advantage of sending the same pulse twice through an unknown channel where both pulses are distorted the same way and detection becomes easier with an autocorrelation receiver. However the method uses a single UWB pulse shape for all channels. The scheme proposed by the authors of this paper uses multiple orthogonal pulses for a UWB system and is a step towards combining the multi-pulse approach and TR 
modulation in a multiple access ultra wideband (MA-UWB) communications system.

This paper first summarizes the performance of a multipulse multi-delay (MPMD) modulation scheme [3]. The pulses used are mutually orthogonal chirp pulses. The idea of using chirp signals for communications was first introduced by Winkler [10]. Chirp signals perform well at the presence of fading due to multipath and have been used in high frequency data transmission applications extensively [12]. El-Khamy et al. also proposed the use of chirp signals for efficient multiple access communications in [13]. Section 2 describes the basic parameters for the waveform, transmitter, and receiver design for MPMD modulation technique. Section 3 provides a summary of the results. Finally in Section 4, some possible new ways of using leastsquares filtering techniques that might be useful in the TR method is discussed.

\section{MPMD MODULATION}

The basic idea is to use multiple mutually orthogonal TR modulated pulses for multiple-access. A symbol in TR modulation consists of two similar pulses separated by a delay $(D)$. The first pulse is fixed and called reference pulse (ref) and the second pulse is modulated with data called data pulse (data). The data pulse modulation scheme is based on the polarity of these pulses. For instance a reference and a data pulse of the same polarity designates a binary value of 1 , while a data pulse opposite in polarity with reference pulse designates a binary value of 0 . Assuming a uniform symbol period, the general signal model of a MPMD system with $N$ users can be expressed as

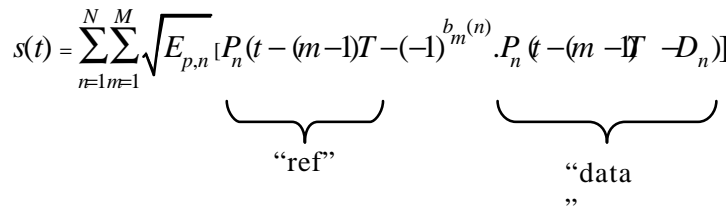

where $N=$ Number of users, $M=$ Number of transmitted bits, $E_{p, n}=\mathrm{n}^{\text {th }}$ user's signal energy (normalized for all users), $P_{n}(t)$ $=\mathrm{n}^{\text {th }}$ user's UWB pulse, $b_{m}{ }^{(n)}=\left[\mathrm{b}_{1}{ }^{(\mathrm{n})}, \ldots, \mathrm{b}_{\mathrm{M}}{ }^{(\mathrm{n})}\right] \mathrm{n}^{\text {th }}$ user's $\mathrm{m}^{\text {th }}$ data bit, $\mathrm{b}_{\mathrm{m}}{ }^{(\mathrm{n})} \in[0,1], D_{n}=\mathrm{n}^{\text {th }}$ user's delay, and $T=$ Pulse repetition period. The UWB pulses used can be short duration chirp pulses with different start and end frequencies.

MPMD receiver uses the following facts to recover the UWB pulses from various channel distortions due to multipath, fading, and noise.

1) The shape of UWB pulses can be considerably degraded due to channel distortions, while the shape of their ACF is relatively preserved at the receiver.

2) Wideband pulses can be designed to have autocorrelation functions with strong side lobes.
3) Information stored in ACF side lobes is additional valuable information that can provide significant performance improvement in detection of multiple pulse systems.

MPMD receiver samples the ACF of each user's pulse at both zero- and non-zero lags. These samples are then matched to the corresponding samples taken from ACF of transmitted pulses, rather than sampling and matching the pulse shape. The input signal at the receiver is given by

$$
r(t)=s(t)+w(t)
$$

where $s(t)$ is the combined signal for $N$ users with $M$ data bits from (1) and $w(t)$ is AWGN with zero mean and twosided power spectral density $N_{0} / 2$.

The proposed demodulation scheme has two steps. Step one samples the shape of the received signal's ACF at multiple points. The sampling is achieved from multiplying the received signal by its multiple delayed versions. The second step involves matching the sampled values from previous step to the sampled values of original pulses' ACFs. Note that matching is performed over the second order statistics, such as the ACF, and not on the signal shape, as is conventionally performed in classical matched filters. Fig. 1 represents the MPMD receiver block diagram.

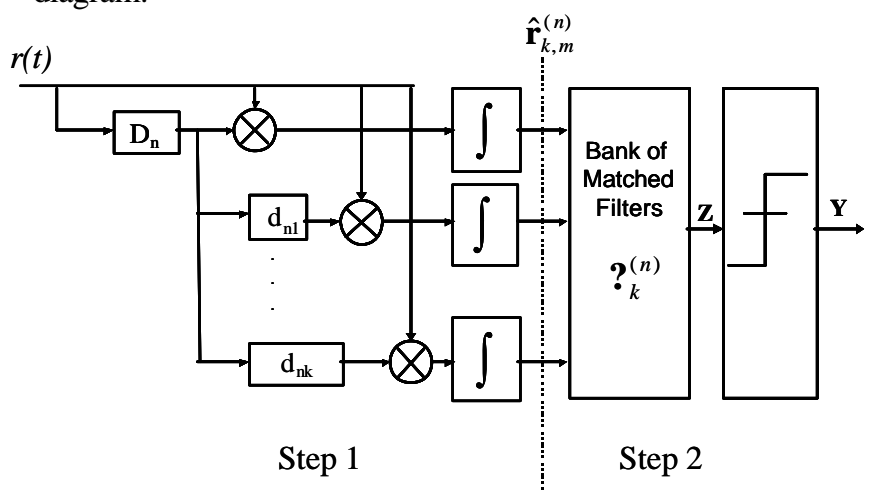

Fig. 1. MPMD Receiver Block Diagram

As shown in Fig.1 the receiver employs autocorrelation technique by multiplying the received signal with multiple delayed versions of itself and integrating over a finite time. Then a bank of matched filters matched to ACF samples of original pulses at transmit stage $\left(\boldsymbol{?}_{k}^{(n)}\right)$, followed by a hard decision block separates each channel. Note that $D_{n}$ and $d_{n}$ are unique for each receiver channel. The output of the receiver is

$$
\mathbf{Y}=\underbrace{\operatorname{sgn}\left(\boldsymbol{?}_{k}^{(n)} \cdot \hat{\mathbf{r}}_{j, m}^{(n)}\right)}
$$

where

\section{$\mathbf{Z}$}

$$
\boldsymbol{?}_{k}^{(n)}=\left[\begin{array}{lllll}
R_{p_{n} p_{n}}^{(n)}(1) & \cdot & \cdot & R_{p_{n} p_{n}}^{(n)}(K)
\end{array}\right]
$$




$$
\hat{\mathbf{r}}_{k, m}^{(n)}=\left[\begin{array}{ccccc}
\hat{r}_{(1,1)}^{(n)} & \cdot & \cdot & \cdot & \hat{r}_{(1, m)}^{(n)} \\
\cdot & \cdot & & & \cdot \\
\cdot & & \cdot & & \cdot \\
\cdot & & & \cdot & \cdot \\
\hat{r}_{(K, 1)}^{(n)} & \cdot & \cdot & \cdot & \hat{r}_{(K, m)}^{(n)}
\end{array}\right]
$$

$\boldsymbol{?}_{k}^{(n)}$ denotes the normalized autocorrelation vector of $\mathrm{n}^{\text {th }}$ user's transmitted pulse for k sampling points (lags). $\hat{\mathbf{r}}_{k, m}^{(n)}$ is the autocorrelation matrix of $\mathrm{n}^{\text {th }}$ user's $\mathrm{m}^{\text {th }}$ bit for $\mathrm{k}$ lags which represents the estimated ACF sampled points at the receiver. Each element of the matrix in (5) represents the autocorrelation between the received signal and its delayed version based on various delays as shown in (6).

$$
\hat{r}_{k, m}^{n}=\int_{(m-1) T}^{(m-1) T+T_{i n}} r(t) r\left(t-\Delta_{n}\right) d t
$$

where $T_{i n}$ is the integration time and $\Delta_{n}$ is the total delay as

$$
\Delta_{n}=D_{n}+d_{n k}
$$

$D_{n}$ represents the $\mathrm{n}^{\text {th }}$ users main delay that provides lag zero in ACF. $d_{n k}$ denotes the offset from its main delay or lag $k$ in $\mathrm{ACF}$ and its value is zero for $k=1\left(d_{n l}=0\right)$. Delaying the received signal by $D_{n}$ causes the "ref" pulse to align with the "data" pulse in each symbol. The product of these aligned pulses followed by integration over a finite time decodes the symbols for received data bits by capturing the energy in lag zero of the generated ACF. This product generates positive values when the symbol represents a binary 1 , and negative values for a binary 0 . Further delaying the received signal by multiple offsets $\left(d_{n k}\right)$ added to the main delay $\left.\nabla_{n}\right)$ and multiplying with its un-delayed version, samples the autocorrelation function in non-zero lags after integration. The sampled points provide estimates of the received signals' ACF shape. These estimated values $\left(\hat{\mathbf{r}}_{k, m}^{(n)}\right)$ are then matched to the sampled values of the original pulses' ACF $\left(\boldsymbol{?}_{k}^{(n)}\right)$ and provide a more accurate decoding of the received symbols. Fig. 2 illustrates matching of ACF samples in a transmitted and received pulse.

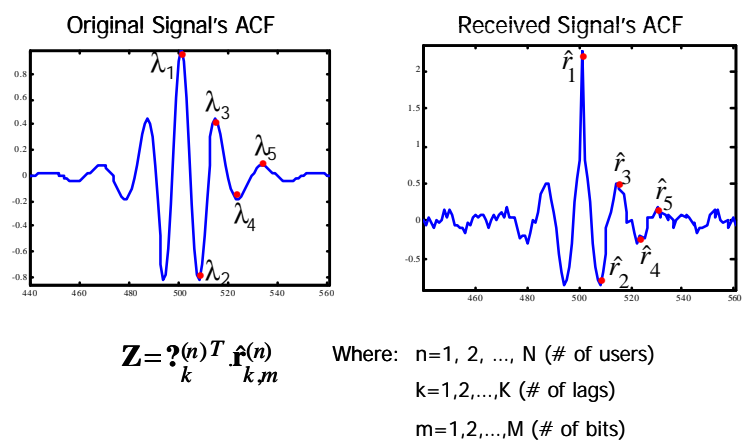

Fig.2. Matching the Shape of ACFs for a Transmitted and Received Pulse

\section{MPMD PERFORMANCE}

In this section the performance of a MPMD system is analyzed based on multiple delays and multiple autocorrelation sampling points. All bit error rate simulations in this section were carried out with total number of 100,000 bits per data point. Fig.3 represents the performance improvement by increasing the number of sampling points $(k)$ in the received signal's ACF. Higher number of sampling points in ACF of the received signal provides more accuracy in the match filtering process and results in BER improvements of the system.

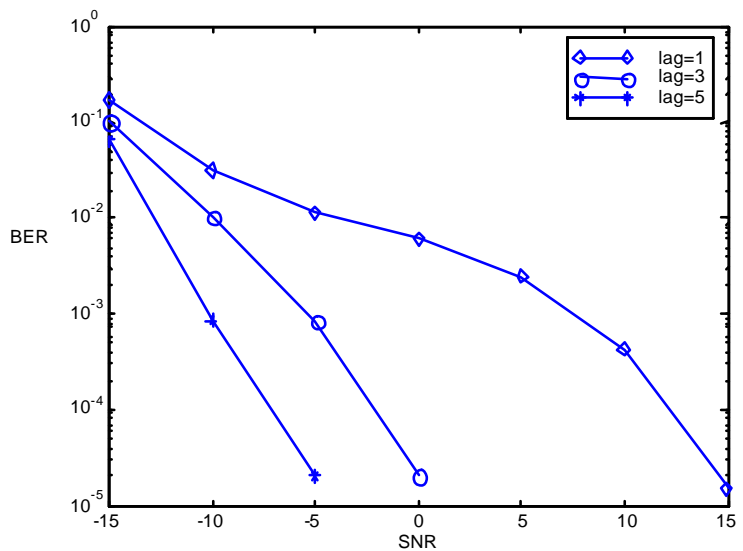

Fig. 3. BER Versus ACF Sampling Points $(K)$ for 10 Users With $1 \mathrm{~ns}$ Difference Between Each Users' Pulse Pair $\left(D_{n}-D_{n-1}=1 n s\right)$.

This modulation scheme is also very effective in multipath environments. Since the same pulse is sent twice through a channel where both pulses are distorted the same way by multipath. Therefore the autocorrelation of the distorted "ref" pulse and distorted "data" pulse can still recover the transmitted signal.

\section{Performance Improvement With Least Squares Filtering Technique}

In this section we consider the problem of filtering the pulse pair in the TR method by using well-known leastsquares filtering techniques. Since in the TR method we are looking for the correlation between a pair of pulses, a filtering technique that would be appropriate would consist of a filter that would pass that component of a two-channel signal which is common to both channels, both in the transmit and the reference channels. Hence, the filtering problem at the receiver after the delay has been removed looks as follows

$$
\begin{aligned}
& x_{1}(t)=s(t)+n_{1}(t) \\
& x_{2}(t)=s(t)+n_{2}(t)
\end{aligned}
$$


where $s(t)$ is the signal and the noise or interference components are $\left\{n_{1}(\eta), n_{2}(t)\right\}$. The filtered signal whose autocorrelation function is sought in the TR receiver is defined to be the sum of the separate outputs of two filters:

$$
y(t)=h_{1}(t) * x_{1}(t)+h_{2}(t) * x_{2}(t)
$$

and the filters $\left\{h_{1}(t), h_{2}(t)\right\}$ are constructed such that:

$$
y(t)=s(t)+\left\{\text { termsof } n_{1}(t) \operatorname{and}_{2}(t)\right\}
$$

The LS filter that minimizes the output energy while passing the signal can be expected to filter out the uncorrelated components $\left\{n_{1}(t), n_{2}(t)\right\}$. Such a filter can be constructed by the Lagrange's method [15], where the constraints on the corresponding discrete-time filter coefficients can be shown to be:

$$
\left\{h_{1}(0)+h_{2}(0)=1, h_{1}(1)+h_{2}(1)=0, \cdots, h_{1}(L)+h_{2}(L)=0\right\}
$$

In fact, the filter coefficients can be efficiently solved by the following equations:

$$
\underline{h}=R^{-1} G\left(G^{T} R^{-1} G\right)^{-1} D
$$

where (taking a $2^{\text {nd }}$ order filter example)

$$
\underline{h}=\left[\underline{h}(0) h_{2}(0) h_{1}(1) h_{2}(1) h_{1}(2) h_{2}(2)\right]
$$

and

$$
G=\left[\begin{array}{llllll}
1 & 1 & 0 & 0 & 0 & 0 \\
0 & 0 & 1 & 1 & 0 & 0 \\
0 & 0 & 0 & 0 & 1 & 1
\end{array}\right], D=\left[\begin{array}{l}
1 \\
0 \\
0
\end{array}\right]
$$

and $\mathrm{R}$ is block Toeplitz covariance matrix from the transmit and reference pulses:

$$
R=\left[\begin{array}{cccccc}
r_{t t}(0) & r_{t r}(0) & r_{t t}(-1) & r_{t r}(-1) & r_{t t}(-2) & r_{t r}(-2) \\
r_{r t}(0) & r_{t t}(0) & r_{t r}(-1) & r_{t t}(-1) & r_{t r}(-2) & r_{t t}(-2) \\
r_{t t}(1) & r_{t r}(1) & r_{t t}(0) & r_{t r}(0) & r_{t t}(-1) & r_{t r}(-1) \\
r_{r t}(1) & r_{r r}(1) & r_{r t}(0) & r_{r r}(0) & r_{r t}(-1) & r_{r r}(-1) \\
r_{t t}(2) & r_{t r}(2) & r_{t t}(1) & r_{t r}(1) & r_{t t}(0) & r_{t r}(0) \\
r_{r t}(2) & r_{r r}(2) & r_{r t}(1) & r_{r r}(1) & r_{r t}(0) & r_{r r}(0)
\end{array}\right]
$$

where the entries are the covariance lags between the transmit and reference pulses denoted by subscripts $r$ and $t$, respectively.

In summary, we are proposing that the TR-UWB scheme might benefit for constrained optimum filtering algorithms. We show this by taking a particular filter design method discussed above. Of course the cost of this is that the computation burden is increased for the overall receiver design.

\section{Conclusions}

The MPMD multiple access method proposed previously seem to provide high bit error rate performance of a UWB multiple access system in low SNR channels. However, any method must eventually deal with noise and interference. We have discussed how well-known methods of least-squares optimal filtering might be used to improve receiver performance.

\section{REFERENCES}

[1] www.fcc.gov

[2] J. Foerster, E. Green, S. Somayazulu, D. Leeper, "Ultra Wideband Technology for Short -or Medium-Range Wireless Communications", Intel Technology Journal Q2, 2001.

[3] F. Nekoogar, F. Dowla, "Multiple Access in Ultra-Wideband Communications Using Multiple Pulses", Wireless Data Communications Onboard Spacecraft-Technology and Applications Workshop” ESA/ESTEC, Noordwijk, NL, April 1416, 2003.

[4] M.Z. Win and R. A. Scholtz, "Ultra-wide Bandwidth TimeHopping Spread Spectrum Impulse Radio for Wireless Multiple Access Communications", IEEE Transactions on Communications, vol. 48, pp., 679-691, Apr. 2000

[5] C.J. Le Martret, G.B. Giannakis, "All-digital PAM impulse radio for multiple-access through frequency-selective multipath" Global Telecommunications Conference, 2000. GLOBECOM '00.

[6] L. Zhao, A. M. Haimovich, " Capacity of M-ary PPM Ultra Wideband Communications over AWGN Channels", IEEE Vehicular Technology Conference, 2001.

[7] J.D. Choi, W. E. Stark, "Performance of Autocorrelation Receivers for Ultra-Wideband communications with PPM in Multipath Channels", IEEE Conference on Ultra Wideband Systems and Technologies, 2002.

[8] A. Spiridon, F. Dowla, T. Rosenbury, and D. Benzel, " Performance of Ultra-Wideband Differential Pulse Waveform", Internal publication at Lawrence Livermore National Laboratory, Feb. 2001.

[9] R. Hoctor, H. Tomlinson, "Delay-hopped TransmittedReference RF Communications", 2002 IEEE Conference on Ultra Wideband Systems and Technologies, May 2002.

[10] M. Ghavami, L. B. Michael, R. Kohono, " Hermite Function Based Orthogonal Pulses for Ultra Wideband Communications", 2002 IEEE Conference on Ultra Wideband Systems and Technologies, May 2002.

[11] M.R. Winkler, "Chirp Signals for Communication", IEEE Wescon Conc. 1962.

[12] G. F. Gott and J. P. Newsome, "H.F. Data Transmission Using Chirp Signals", Proc. of IEE., vol. 118, pp. 1162-1166, Sept. 1971.

[13] S.E.El-Khamy, S.E.Shaaban, E.A.Thabet, "Multiuser Chirp Modulation Signals (M-CM0 for Efficient Multiple Access Communication Systems", Radio Science Conference, March 1996.

[14] J. G. Proakis, Digital Communications, Third Eddition, McGrawHill, Inc., New York, 1995. 
[15] J. F. Clarebout, "A Summary, by Illustrations, of Least-Squares Filters with Constraints,", IEEE Trans. Information Theory, Vol.-IT-14, No. 2, pp. 269-272, March 1966. 